\title{
ASPECTOS ERGONÔMICOS E ESTATÍSTICOS NO PROJETO DE UM CARRO DO METRÔ
}

\section{GESTÃO \\ $\&$ \\ PRODUÇÃO}

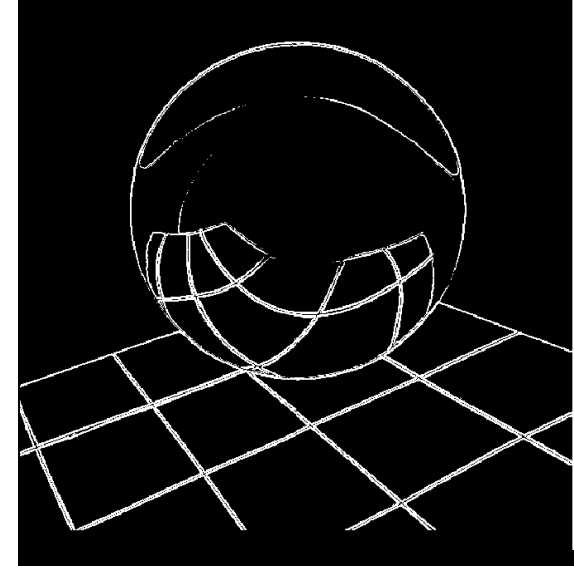

v.9, n.1, p.93-105, abr. 2002

\author{
Pedro Luiz de Oliveira Costa Neto \\ Escola Politécnica da USP \\ UNIP - Universidade Paulista \\ Faculdades Tancredo Neves \\ São Paulo - SP \\ E-mail: politeleia@uol.com.br
}

\section{Carlos Maurício Duque dos Santos \\ UNIP - Universidade Paulista \\ DCA Ergonomia \& Design \\ São Paulo - SP \\ E-mail:mauricioduque@uol.com.br}

\section{Resumo}

Este artigo descreve experimentos realizados como parte de um trabalho envolvendo aspectos do projeto de um carro para uma nova linha do Metrô de São Paulo. Nele são considerados aspectos ergonômicos relacionados com a posição de barras de segurança e assentos, bem como aspectos estatísticos relacionados com o fluxo de passageiros entrando e saindo do carro na estação. Nesse estudo, é de particular interesse o uso da técnica do quadrado latino na análise de regressão múltipla, como forma de reduzir o porte do experimento.

Palavras-chave: ergonomia, medidas antropométricas, fluxo de passageiros, quadrado latino, análise de regressão múltipla.

\section{Introdução}

$\mathrm{O}$ presente artigo sumariza uma experiência realizada em 1989 como parte de um projeto desenvolvido pelo GAPP - Grupo Associado de Pesquisa e Planejamento Ltda. para a Companhia do Metropolitano de São Paulo. A defasagem no tempo, entretanto, a nosso ver, não diminui a atualidade dos assuntos discutidos que, por diversas razões, não foram publicados anteriormente. De fato, o que ensejou a publicação deste artigo apenas agora foi o fato de seus autores haverem se reencontrado, exercendo atividades docentes na Universidade Paulista.

O contexto da experiência antecedeu a tomada de decisões quanto ao projeto definitivo dos 
carros do Metrô que iriam operar no ramal da Avenida Paulista. Testaram-se, então, comparativamente, duas possíveis configurações, com três ou quatro portas. Na primeira delas, mais ampla, se estudava a colocação ou não de um pegador central em frente à porta.

Foi realizada uma simulação da condição real de operação de um carro do metrô por meio da utilização de um mock-up, correspondendo a uma fração do carro em tamanho real. Esta fração correspondia a $1 / 3$ ou $1 / 4$, conforme o caso simulado fosse de um carro com 3 ou 4 portas. Funcionários da Companhia do Metropolitano colaboraram com o experimento, simulando os usuários reais do sistema.

A experiência tem aspectos ergonômicos e estatísticos que julgamos interessante relatar. No aspecto estatístico, cremos interessar o uso que se fez da técnica de "quadrado latino" aplicada em regressão, como forma de reduzir o porte do experimento.

\section{Aspectos Ergonômicos dos Testes Estáticos e Dinâmicos}

$\mathrm{O}$ estudo em questão teve como objetivo a otimização de soluções projetuais relativas à configuração do salão de passageiros no que diz respeito ao lay-out de bancos e pegadores (facilitação de posicionamento e movimentação de usuários no interior dos carros) e no que se refere à localização e dimensionamento da largura das portas (facilitação de entrada e saída de passageiros dos carros) visando, objetivamente, a diminuição dos tempos de embarque e de vazão de usuários nas estações.

Para a conveniente realização dos estudos e experimentos realizados no presente projeto, recorreu-se aos princípios e recomendações existentes na literatura, com destaque para BONSIEPE (1978), COUTO (1966), DIFFRENT et al. (1975), PANERO \& ZELNIK (1979) e WISNER (1987).

Os referidos estudos foram realizados por meio de diversos testes ergonômicos funcionais efetuados em mock-ups, e os seus resultados contêm as conclusões e recomendações gerais decorrentes dos diversos subsistemas testados, fornecendo subsídios para o prosseguimento do Projeto dos Carros da Linha Paulista do Metrô.

Em todos os estudos e testes realizados, foram levadas em consideração as articulações dos parâmetros testados entre si, de modo a permitir que esses resultados subsidiassem a escolha de conjuntos otimizados de soluções parciais, tendo em conta suas respectivas interfaces, para o projeto definitivo.

Os estudos ergonômicos tiveram como base os testes estáticos e dinâmicos que foram realizados em mock-ups experimentais, construídos especialmente para os estudos.

\subsection{Os Parâmetros dos Testes Ergonômicos Estáticos e Dinâmicos}

Os testes foram realizados levando-se em consideração parâmetros prefixados, baseados em soluções alternativas constantes do lay-out do projeto preliminar dos carros da Linha Paulista, quais sejam:

a) Portas:

- alternativa de carro com três portas com largura de $1870 \mathrm{~mm}$ cada;

- alternativa de carro com quatro portas com largura de $1600 \mathrm{~mm}$ cada.

b) Pegadores:

- pegadores horizontais laterais duplos, pegador longitudinal central contínuo, colunas verticais dos pegadores horizontais, pegadores horizontais (baixos) junto às portas e coluna vertical central entre portas.

c) Bancos:

- transversais e longitudinais.

Devido à natureza e à peculiaridade dos parâmetros testados, os testes ergonômico-funcionais foram divididos em estáticos e dinâmicos.

Importa destacar, em relação aos testes de vazão, que foram realizados em caráter comparativo entre as alternativas do carro com três portas e carro com quatro portas, fixando-se 
como opção de escolha a alternativa de melhor desempenho operacional, ou seja, em termos de menor gasto de tempo no conjunto de testes efetuados.

\subsection{Os Testes Estáticos}

Os testes estáticos foram assim denominados por envolverem parâmetros que não exigiam a movimentação de usuários, abrangendo os seguintes subsistemas:

- localização, posicionamento e dimensionamento de alturas relativas ao alcance físico aos pegadores (horizontais e verticais) por parte dos usuários;

- densidade de passageiros no interior do vagão em relação ao posicionamento de bancos e pegadores;

- lay-out dos bancos transversais e longitudinais, em relação à situação de acesso de usuários aos bancos transversais encostados na lateral do vagão.

Foram realizados nove tipos de testes, com usuários de estaturas variando de $1,50 \mathrm{~m}$ a $1,95 \mathrm{~m}$ e de diferentes tipos físicos (endomorfo, ectomorfo e mesomorfo).

Todos os testes foram realizados em mock-up construído especialmente para a finalidade. A seguir, listamos os testes estáticos realizados, apenas para demonstrar a abrangência do estudo em questão:

1. posição e altura do pegador longitudinal central;

2. alcance do pegador longitudinal central;

3. alcance do pegador horizontal lateral superior;

4. alcance do pegador horizontal lateral inferior;

5. densidade de usuários em linha (seção transversal do vagão);

6. determinação da distância entre pegadores horizontais laterais;

7. determinação da distância entre bancos, para acesso de usuários;

8. acesso e acomodação de usuários do banco transversal;
9. determinação das dimensões básicas do banco para o usuário.

Os resultados dos testes estáticos permitiram determinar com exatidão o dimensionamento e posicionamento de bancos e pegadores, de forma a proporcionar melhor conforto para os usuários.

Descrevemos, a seguir, os conceitos e funções do mock-up, dos dados antropométricos e sujeitos dos testes, bem como os critérios dos testes estáticos.

\subsubsection{O Mock-up}

Para a realização dos testes estáticos foi construído um mock-up de trecho representativo do carro, reproduzindo as condições necessárias para a execução dos testes. Trata-se de uma ferramenta de projeto utilizada para testes dimensionais, estudo de volumes, configurações físicas, lay-outs e, até mesmo, aspectos estético-formais.

O mock-up é normalmente construído em escala 1:1 e com materiais de baixo custo e grande flexibilidade, de forma a proporcionar meios para alterações rápidas. Uma visualização do tipo de mock-up usado no presente estudo pode ser obtida nas Figuras 1 e 2.

\subsubsection{Dados Antropométricos e Sujeitos dos Testes}

Para a realização dos testes estáticos, foram utilizados dados antropométricos brasileiros constantes na publicação MCT (1988). Os sujeitos dos testes foram selecionados de acordo com os percentis antropométricos (variando de $5 \%$ a 95\%), totalizando 15 sujeitos, que constituíram uma amostra suficiente para os objetivos desejados, por incorporar pessoas com características físicas diversificadas

Para os testes de determinação e verificação de alcances físicos de pegadores e posicionamento e acomodação em bancos, bem como para a determinação de medidas básicas do banco de passageiros, foram utilizados dados antropométricos pertinentes a cada situação. Assim, para os alcances e acomodação foram utilizados 


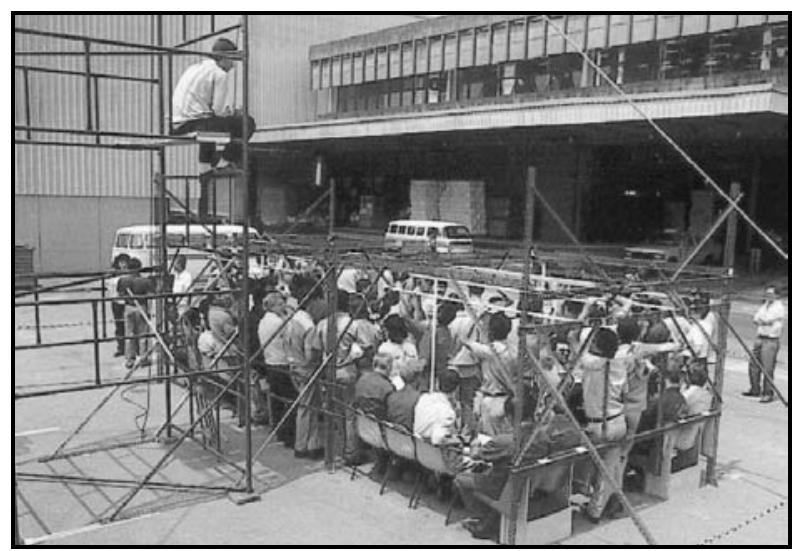

Figura 1 - Vista geral do mock-up

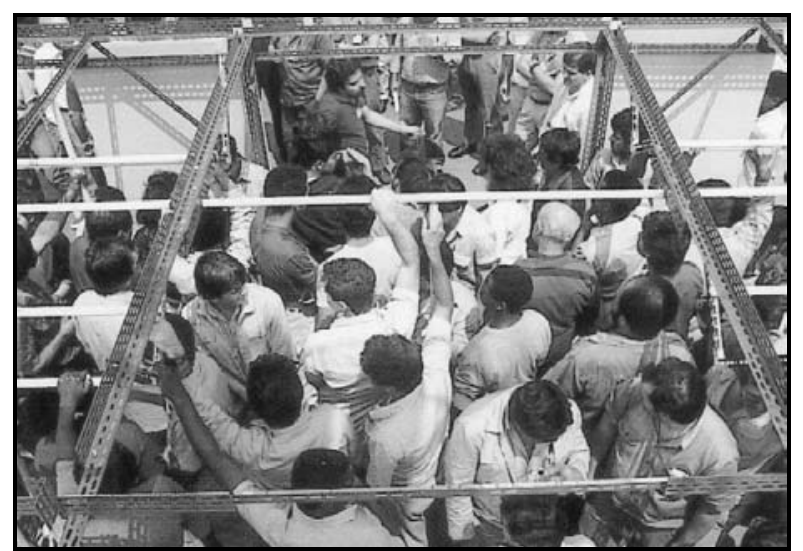

Figura 2 - Mock-up ocupado por passageiros em movimento

percentis de estaturas e, para as medidas básicas do banco, foram utilizados percentis relativos à altura popliteal, profundidade nádega-popliteal e profundidade nádega-joelho.

A seleção dos sujeitos para os testes foi feita sempre de modo a se adequarem à natureza de cada teste, em função dos valores atribuídos ou determinados pelos percentis tabelados. Para os testes de distância entre pegadores laterais e de densidade, foram selecionados aleatoriamente sujeitos de percentis diversos, porém com biótipos considerados normais.

\subsubsection{Critérios Gerais de Execução dos Testes}

Para todos os testes efetuados, foram estabelecidos três níveis de avaliação, a saber:
- Adequado: este nível significa que o esforço que o usuário despende para cumprir o teste (postura, alcance físico, acomodação, acesso, etc.) está plenamente dentro dos limites de conforto considerados normais.

- Suficiente: este nível significa que, apesar de o usuário não estar dentro dos limites de conforto considerados bons, ainda assim o seu esforço pode ser considerado aceitável, visto que ele ainda consegue realizar a ação desejada.

- Inadequado: este nível significa que as condições exigidas do usuário para realizar suas ações são totalmente fora das condições de conforto e, até mesmo, inexeqüíveis fisicamente. 


\subsubsection{Resultados dos Testes Estáticos}

A título de ilustração, apresentamos a seguir os resultados dos testes estáticos:

a. Altura do pegador longitudinal central $(202 \mathrm{~cm})$ :

- Adequado: indivíduos com estatura menor que $187 \mathrm{~cm}$;

- Suficiente: indivíduos com estatura entre $187 \mathrm{~cm}$ e $200 \mathrm{~cm}$;

- Inadequado: indivíduos com estatura superior a $200 \mathrm{~cm}$.

b. Alcance com as mãos no pegador longitudinal central $(204 \mathrm{~cm})$ :

- Adequado: indivíduos com estatura maior ou igual a $187 \mathrm{~cm}$;

- Suficiente: indivíduos com estatura entre $170 \mathrm{~cm}$ e $187 \mathrm{~cm}$;

- Inadequado: indivíduos com estatura inferior a $170 \mathrm{~cm}$.

c. Alcance com as mãos no pegador horizontal superior $(195 \mathrm{~cm})$ :

- Adequado: indivíduos com estatura maior ou igual a $173 \mathrm{~cm}$;

- Suficiente: indivíduos com estatura entre $165 \mathrm{~cm}$ e $173 \mathrm{~cm}$;

- Inadequado: indivíduos com estatura inferior a $165 \mathrm{~cm}$.

d. Alcance com as mãos no pegador horizontal inferior $(183 \mathrm{~cm})$ :

- Adequado: indivíduos com estatura maior ou igual a $165 \mathrm{~cm}$;

- Suficiente: indivíduos com estatura entre $158 \mathrm{~cm}$ e $165 \mathrm{~cm}$;

- Inadequado: indivíduos com estatura inferior a $158 \mathrm{~cm}$.

e. Densidade de usuários em linha (seção transversal do vagão):

- Adequado: até 5 indivíduos;

- Suficiente: 6 ou 7 indivíduos;

- Inadequado: acima de 7 indivíduos.

f. Determinação da distância entre pegadores horizontais laterais:

- Adequado: maior que $21 \mathrm{~cm}$;

- Suficiente: entre $17 \mathrm{~cm}$ e $21 \mathrm{~cm}$;

- Inadequado: menor que $17 \mathrm{~cm}$. g. Determinação da distância entre bancos para acesso de usuários (percentil $99=187 \mathrm{~cm}$ ):

- Adequado: maior ou igual a $33 \mathrm{~cm}$;

- Suficiente: entre $28 \mathrm{~cm}$ e $33 \mathrm{~cm}$;

- Inadequado: menor que $28 \mathrm{~cm}$.

h. Acesso e acomodação de usuários do banco transversal:

- Adequado: maior ou igual a $33 \mathrm{~cm}$;

- Suficiente: entre $28 \mathrm{~cm}$ e $33 \mathrm{~cm}$;

- Inadequado: menor que $28 \mathrm{~cm}$.

i. Determinação das dimensões básicas do banco do usuário:

- Profundidade do assento: $37 \mathrm{~cm}$;

- Altura do encosto: $47 \mathrm{~cm}$;

- Ângulo entre assento e encosto: $100^{\circ}$;

- Altura da borda superior do assento: 39 $\mathrm{cm}$ (altura popliteal, percentil 5\%, indivíduo com estatura de $159 \mathrm{~cm}$ );

- Ângulo de inclinação do assento em relação à horizontal: $5^{\circ}$.

\section{Os Testes Dinâmicos}

Os testes dinâmicos foram realizados em mock-ups com trechos representativos equivalentes a 1/3 do carro, para a alternativa de carro com 3 portas, e a 1/4 do carro, para a alternativa de carro com 4 portas. Com o objetivo de conseguir dados comparativos entre estas duas opções, todos os testes foram realizados para ambas alternativas.

Os testes dinâmicos abrangeram estudos relativos às seguintes condições:

- Lotação do carro: com quantidades de usuários diversas, em função de cada situação testada;

- Quantidade de usuários na plataforma: entrando no carro com quantidades diversas, em função de cada situação testada;

- Usuários saindo do carro: com quantidades de usuários diversas, em função de cada situação testada.

A seleção e determinação de cada experiência, assim como a quantificação de usuários necessários para cumprir cada um dos testes, foram objeto de estudos estatísticos de regressão 
incorporando-se a técnica do quadrado latino, conforme mostrado no item 5.2.

Alguns dos testes realizados foram também acrescidos de outras variáveis, como a imposição, em metade dos casos, da utilização de pegador vertical no centro do espaço entre as duas portas frontais, bem como o posicionamento de usuários postados em lugares inconvenientes para o processo de fluxo normal de embarque/desembarque, como, por exemplo, junto aos batentes das portas.

Além destes fatores, foi agregada aos testes a determinação de fluxos de usuários em intervalos de tempos variáveis nas operações de embarque/desembarque, obtidos através da contagem de tempo na gravação em videotape.

\subsection{População Utilizada nos Testes Dinâmicos}

Para a realização dos testes, foram utilizadas diversas pessoas (funcionários da Cia. do Metrô, pertencentes ao Pátio de Jabaquara, homens e mulheres adultos e adolescentes), as quais foram consideradas como representativas da população de usuários que, em condições reais de operação, se utilizariam do transporte oferecido pelos trens do Metrô.

A fim de testar, nas condições práticas, a capacidade de um carro, conduziu-se inicialmente uma experiência que constou da simples lotação básica do mock-up com elementos da amostra, o que levou a uma estimativa para a lotação básica do mock-up (correspondente a 1/3 do carro) de 115 pessoas, indicando que o carro tem capacidade para transportar 345 pessoas. Este número passou a ser adotado como base para os testes de fluxo.

\subsection{Resultados dos Testes Dinâmicos}

A experiência foi suficiente para demonstrar que o tempo médio necessário para a entrada e saída de passageiros é significativamente menor para a configuração de 4 portas de $1600 \mathrm{~mm}$ que para aquela com 3 portas de $1870 \mathrm{~mm}$. Isso confirmou, de certa forma, o esperado, pois
$4 \times 1600=6400 \mathrm{~mm}$ representa uma abertura total para escoamento maior que $3 \times 1870=$ $5610 \mathrm{~mm}$.

Outros resultados foram obtidos através de análise estatística dos dados, uma parte da qual é reproduzida a seguir. Nesse estudo, acreditamos ter particular interesse a forma como se procurou reduzir o tamanho do experimento utilizando-se a técnica do quadrado latino, visando tornar a experiência viável. Para melhor compreensão do que foi feito, apresenta-se a seguir uma introdução a essa técnica.

\section{A Técnica do Quadrado Latino}

$\mathrm{E}$ m um delineamento de experimento fatorial, existem alguns fatores (causas) sendo investigados, cada um com um certo número de possíveis níveis, e uma resposta sendo medida para cada combinação dos níveis de fatores (condições experimentais).

O experimento será completo se todas as condições experimentais forem investigadas, totalizando um número de situações igual ao produto dos números de níveis de cada fator. Se o número de níveis for igual para todos os fatores, a técnica de quadrado latino pode eventualmente ser usada para reduzir consideravelmente o número de condições experimentais.

Ilustremos isso supondo que haja três fatores I, II e III, cada um envolvendo quatro níveis. Em um fatorial completo haverá, portanto, $4 \times 4 \times 4=64$ condições experimentais a investigar. Mediante um quadrado latino, este número poderá ser reduzido a 16, usando-se, por exemplo, a configuração apresentada na Tabela 1 , em que as letras no corpo da tabela representam os quatro níveis possíveis do fator III. Assim, são investigadas $25 \%$ das possíveis condições experimentais, segundo uma distribuição harmoniosa, visando a menor perda de informação.

Deve-se notar que a configuração apresentada é apenas uma das possíveis no caso. Haveria cinco outras maneiras de se construir quadrados latinos no presente exemplo. Para maiores informações veja-se, por exemplo, MONTGOMERY (1991). 
Tabela 1 - Quadrado latino 4 X 4

\begin{tabular}{|c|c|c|c|c|}
\hline II & 1 & 2 & 3 & 4 \\
\hline 1 & A & B & C & D \\
\hline 2 & D & A & B & C \\
\hline 3 & C & D & A & B \\
\hline 4 & B & C & D & A \\
\hline
\end{tabular}

Tabela 2 - Quadrado latino 3 X 3

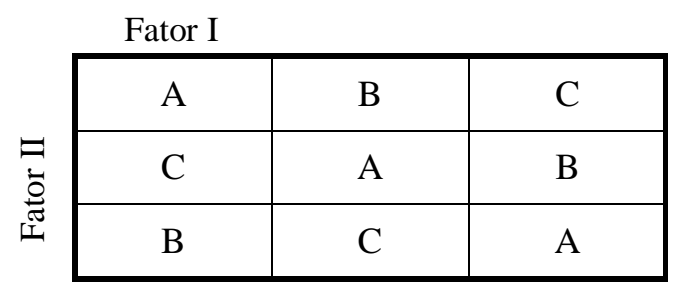

Evidentemente, existe aí uma compensação entre ganho em custo do experimento e perda de poder estatístico da análise. De fato, se, no exemplo, se evita investigar $75 \%$ das possíveis condições experimentais, perdem-se graus de liberdade do resíduo na análise de variância conduzida para testar a significância da influência de cada fator na resposta. Há também a se considerar a perda da possibilidade de se investigar a existência de possíveis interações entre os fatores, supostas desprezíveis neste modelo.

Ilustremos figurativamente, através do quadrado latino dado na Tabela 2, um caso com três níveis.

O cubo da Figura 3 mostra, com os pontos escuros, as 27 combinações de níveis de fator que corresponderiam à realização de um experimento fatorial completo e, realçadas, as nove combinações que correspondem às condições experimentais quando se utiliza o quadrado latino acima.
Considerações estatísticas à parte, que julgamos fora do escopo deste artigo, os quadrados latinos, muitas vezes, podem ser usados como elementos simplificadores do experimento, possivelmente, até, viabilizando-o economicamente.

\section{Aspectos Estatísticos dos Testes Dinâmicos de Fluxo}

Dara estes testes, nos quais diversas condições seriam testadas, envolvendo algumas variáveis de projeto, utilizou-se o mock-up descrito em 2.2.1, correspondente à terça ou à quarta parte de um carro real. Entretanto, embora utilizado para as duas configurações de portas, apresentamos o procedimento apenas no caso de três portas, por ser de interesse mais geral, devido ao uso de uma variável fictícia.

Conforme já visto, tomaram-se 115 pessoas como sendo a lotação máxima para este mock-up. A esta quantidade considerada básica, 


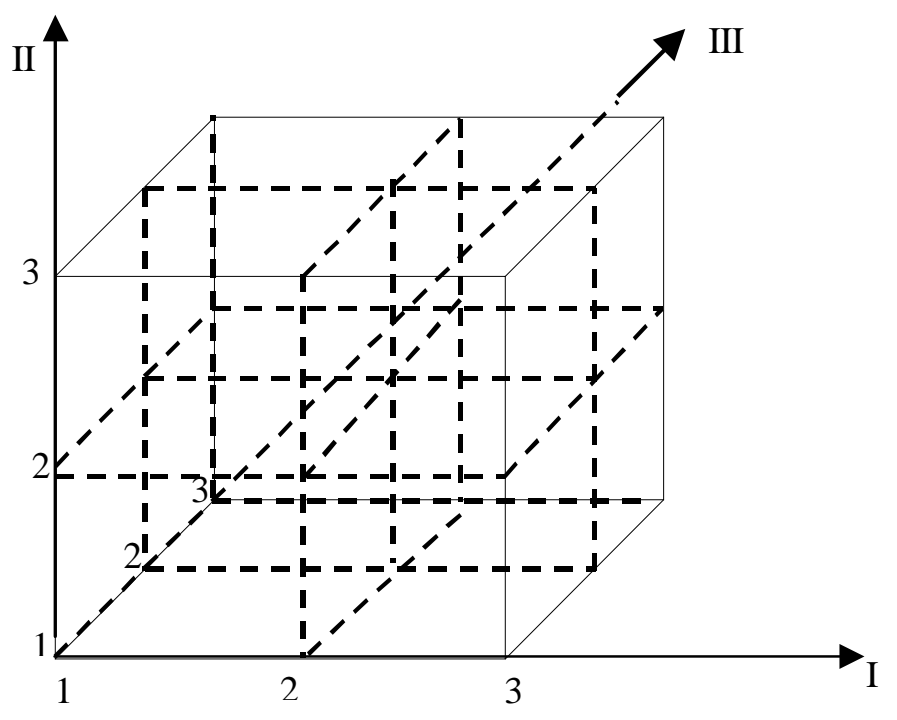

Figura 3 - Representação espacial de um experimento fatorial

foram adicionadas mais 47 pessoas representativas do maior número de usuários postados na plataforma (hipótese de plataforma mais cheia) que deveriam acessar o carro, totalizando então a quantidade de 162 pessoas, número máximo necessário para a realização dos testes como previsto.

Os testes de fluxo consistiram, basicamente, na medição, através de cronometragem e de gravação em videotape, dos tempos necessários à operação de saída de usuários do interior do carro e de entrada de usuários (postados na plataforma) ao seu interior, em ações simultâneas. Desse modo, foram realizados testes em função de diversas quantidades de usuários, tanto no interior do carro como na plataforma.

Os testes foram registrados por intermédio de fotografias e de gravação de videotape, que tiveram como finalidade, além de ilustrar os experimentos, servir também (no caso das gravações) para análise dos testes e cálculo de tempos.

\subsection{Variáveis Estudadas}

Foram consideradas, para efeito da montagem da experiência e especificação dos testes, as seguintes variáveis:
$\mathbf{X}_{\mathbf{1}}=$ densidade de passageiros no carro. Esta variável, originalmente expressa em porcentagem, foi, em cada caso, explicitada em termos de número de usuários no "mock-up" conforme a configuração do carro seja de 3 ou 4 portas. Tendo em vista as condições mais críticas de operação que se pretendia investigar, seus valores foram, para efeitos das experiências, considerados aos níveis 50\%, 75\%, 90\% e $100 \%$.

$\mathbf{X}_{2}=$ quantidade de usuários na plataforma, querendo embarcar. Esta variável, originalmente expressa em número de pessoas querendo embarcar por carro, foi, em cada caso, explicitada em número de usuários querendo embarcar por porta. Para efeito das experiências, foram considerados para esta variável os valores originais 20,60 , 100 e 140 .

$\mathbf{X}_{\mathbf{3}}=$ porcentagem de passageiros do carro descendo na estação. Esta variável, originalmente expressa em porcentagem do número de passageiros dentro do carro, foi, em cada caso, explicitada em termos de número de usuários saindo por porta. 
Para efeito das experiências, foram considerados para esta variável os níveis $5 \%$, $15 \%, 25 \%$ e $35 \%$.

$\mathbf{X}_{4}=$ variável fictícia indicativa da ausência ou presença do pegador vertical central entre portas (somente para a alternativa de carro com 3 portas). Esta variável qualitativa assume valor 0 quando da ausência do pegador, e valor 1 quando da sua presença. Desta forma, procurou-se incorporar aos resultados da experiência o efeito da existência ou não do pegador.

$\mathbf{Y}=$ tempo gasto na operação de entrada/saída de usuários. Esta variável dependente, objeto de todos os testes, teve seus valores medidos em segundos.

Considerou-se "tempo gasto na operação" como sendo o tempo medido a partir do instante de abertura da porta até o encerramento da operação, conforme critério do diretor da experiência.

Para efeito de planejamento das experiências, foi definido que o encerramento da operação se daria ao se completar a entrada e saída de passageiros ou ao se verificar, a critério do diretor, a impossibilidade (por travamento) de se prosseguir com o processo. Na realização das experiências, este segundo caso acabou não ocorrendo.

Deve-se notar que os valores das variáveis independentes quantitativas (variáveis de entrada) $\mathrm{X}_{1}, \mathrm{X}_{2}$ e $\mathrm{X}_{3}$ foram escolhidos tendo em vista simular as condições mais críticas de operação com portas abrindo de um só lado do vagão, com base em dados conhecidos a partir de situações reais de operação.

\subsection{Definição do Experimento}

Considerando-se a existência de três variáveis quantitativas com quatro níveis cada e uma variável qualitativa (fictícia) com dois níveis, existiam 128 combinações experimentais a serem investigadas em um experimento completo, o que seria praticamente inviável no presente caso.

Por esta razão, resolveu-se organizar as variáveis $\mathbf{X}_{\mathbf{1}}, \mathbf{X}_{\mathbf{2}}$ e $\mathbf{X}_{\mathbf{3}}$ conforme um delineamento em quadrado latino, com o que se conseguiria racionalmente investigar toda a gama experimental original, sem necessariamente simular todos os casos e com a mínima perda de informações em face da redução do tamanho do experimento. Além disso, os casos referentes à variável $\mathbf{X}_{4}$ foram também racionalmente distribuídos por sobre esse quadrado latino.

Dentre as configurações possíveis, decidiu-se adotar a apresentada na Tabela 3, a ser usada para ambas as condições básicas.

No corpo da tabela, aparecem os valores da variável $\mathrm{X}_{3}$ e os asteriscos indicam os casos em que $\mathrm{X}_{4}=1$ (pegador central entre portas instalado). Dessa forma, o número de testes foi reduzido para 16. Isso equivale a se adotar, para a configuração de condições experimentais a serem utilizadas para as variáveis independentes quantitativas da regressão, um quadrado latino conforme mostrado na Tabela 4.

Note-se que, a rigor, a presença dos asteriscos indica a existência de uma segunda latinização sobrepondo-se à primeira, em termos da variável fictícia.

Explicitando as variáveis $X_{1}, X_{2}$ e $X_{3}$ em termos do número de pessoas necessárias para cada experiência, temos as quantidades mostradas na Tabela 5.

Estes valores foram determinados com base na capacidade do mock-up, estimada conforme o teste de capacidade em 115 para o carro com 3 portas (seriam 86 para o caso do carro com 4 portas).

\subsection{Sistemática de Testes}

A sistemática adotada para os testes buscou, na medida das possibilidades, reproduzir as condições de uso que se supõe ocorrerem na realidade. Evidentemente, diversas pequenas ocorrências de operação real (tais como passageiros com pacote, passageiros distraídos, 
Tabela 3 - Configuração adotada no experimento

\begin{tabular}{|c|c|c|c|c|}
\hline $\mathrm{X}_{2} \mathrm{X}_{1}$ & $50 \%$ & $75 \%$ & $90 \%$ & $100 \%$ \\
\hline 20 & $35 \% *$ & $25 \%$ & $5 \%$ & $15 \% *$ \\
\hline 60 & $25 \% *$ & $15 \%$ & $35 \%$ & $5 \% *$ \\
\hline 100 & $5 \%$ & $35 \% *$ & $15 \% *$ & $25 \%$ \\
\hline 140 & $15 \%$ & $5 \% *$ & $25 \% *$ & $35 \%$ \\
\hline
\end{tabular}

Tabela 4 - Quadrado latino utilizado no experimento

\begin{tabular}{|c|c|c|c|}
\hline $\mathrm{D} \quad *$ & $\mathrm{C}$ & A & $\mathrm{B} \quad *$ \\
\hline $\mathrm{C} *$ & B & $\mathrm{D}$ & A $*$ \\
\hline A & $\mathrm{D} \quad *$ & B $*$ & $\mathrm{C}$ \\
\hline B & A $*$ & $\mathrm{C} *$ & $\mathrm{D}$ \\
\hline
\end{tabular}

Tabela 5 - Números de pessoas necessárias para o experimento

\begin{tabular}{|c|c|c|c|c|}
\hline $\mathrm{X}_{2}$ & 57 & 86 & 103 & 115 \\
\hline 07 & 64 & 93 & 110 & 122 \\
\hline 20 & 77 & 106 & 123 & 135 \\
\hline 33 & 90 & 119 & 136 & 148 \\
\hline 47 & 104 & 133 & 150 & 162 \\
\hline
\end{tabular}

deficientes físicos, etc.) tiveram que ser abstraídas na experiência, por motivos óbvios.

Entretanto, buscou-se a maior proximidade possível com as situações reais. Para tanto, antes de cada experiência, foram tomadas as seguintes providências:
- distribuição de uma circular relatando a importância do experimento e as instruções básicas, para efeito de esclarecimento e motivação;

- solicitação aos participantes que procurassem proceder, durante a experiência, da mesma forma que normalmente o fariam ao usar o Metrô; 
Tabela 6 - Resultados da regressão stepwise

\begin{tabular}{|c|c|c|}
\hline $\begin{array}{c}\text { ORDEM DE } \\
\text { ENTRADA }\end{array}$ & $\mathrm{F}_{\mathrm{OBS}}$ & INFLUÊNCIA \\
\hline $\mathrm{X}_{2}$ & 23,907 & $52,4 \%$ \\
\hline $\mathrm{X}_{1}$ & 5,350 & $25,0 \%$ \\
\hline $\mathrm{X}_{4}$ & 1,358 & $11,2 \%$ \\
\hline $\mathrm{X}_{3}$ & 1,162 & $11,4 \%$ \\
\hline
\end{tabular}

- distribuição de cartões coloridos para caracterizar os passageiros, assim convencionados:

- cartão vermelho: passageiros dentro do carro prosseguindo viagem

- cartão verde: passageiros dentro do carro desejando sair

- cartão amarelo: passageiros na plataforma desejando entrar

- solicitação aos passageiros descendo que se afastassem imediatamente da porta.

\subsection{Realização da Experiência}

Os testes obedeceram, no geral, à seguinte seqüência de procedimentos:

1. o diretor da experiência dava um primeiro sinal para alertar os participantes, dizendo "atenção";

2. instantes após o apito do diretor, abria-se a porta e iniciava-se a cronometragem;

3. ao completar-se a operação, o diretor dava um segundo apito e o cronometrista marcava o tempo. Para efeito da gravação em videotape, fechava-se a porta;

4. os dados da experiência foram devidamente anotados em fichas-resumo, para posterior análise.

$\mathrm{O}$ diretor da experiência foi sempre o mesmo, colocado em posição elevada, de modo a ter visão interna e externa em relação às ações desenvolvidas, conforme se vê na Figura 1.

\subsection{Análise Estatística}

A análise da regressão múltipla, envolvendo as variáveis $\mathrm{X}_{1}, \mathrm{X}_{2}, \mathrm{X}_{3}, \mathrm{X}_{4}$ e $\mathrm{Y}$, foi feita usandose o programa para stepwise regression do software SPSS. Segundo COSTA NETO (2001), a regressão stepwise se caracteriza por um procedimento em que as variáveis independentes $\left(\mathrm{X}_{\mathrm{i}}\right)$ mais bem correlacionadas com $\mathrm{Y}$ vão sendo sucessivamente incluídas no modelo proposto, sendo, a cada inclusão, testado pela análise de variância se aquela inclusão contribui significativamente para melhorar a representação oferecida pelo modelo. Com isso, pode-se ter uma boa idéia da importância relativa que as diversas variáveis têm na explicação do fenômeno.

Por não ser razoável supor a linearidade do modelo, foram feitas várias tentativas buscando identificar funções linearizáveis adequadas à boa representação do fenômeno. Essa pesquisa indicou ser mais adequado um modelo exponencial em todas as variáveis independentes, chegando-se à expressão:

$\mathrm{Y}=6,6100 \cdot 1,0073^{\mathrm{X}} \cdot 1,0068^{\mathrm{x}} \cdot 1,0055^{\mathrm{x}} \cdot 1,1303^{4}$, donde se estima, pelo coeficiente de $\mathrm{X}_{4}$ (variável fictícia), que a influência da existência do pegador central no aumento do tempo de operação é da ordem de $13 \%$.

$\mathrm{O}$ coeficiente de determinação dessa regressão foi $\mathrm{R}^{2}=0,783$, correspondendo a uma razoável representação do fenômeno.

A Tabela 6 resume as informações fornecidas pela regressão stepwise. 
A variável $\mathrm{X}_{2}$ (usuário embarcando) foi altamente significativa e $X_{1}$ (passageiros no carro) significativa com 5\% de significância. As variáveis $\mathrm{X}_{3}$ (passageiros desembarcando) e $\mathrm{X}_{4}$ (existência do pegador central) não são significativas após a consideração das demais. Isso não significa, entretanto, que não se deva propor a eliminação do pegador.

Devemos reconhecer que a adoção da forma exponencial para as variáveis $X_{1}$ e $X_{3}$ também leva a um aparente paradoxo, pois para $X_{2}=X_{3}=0$, o tempo de operação não se anula como, aparentemente, deveria. De fato, observando as equações obtidas, verifica-se que, para fluxo nulo, o tempo de operação converge para algo da ordem de grandeza de 10 segundos, dependendo do valor $X_{1}$. Isso, entretanto, vai de encontro às condições reais de operação do Metrô, em que sempre as portas permanecem abertas algum tempo, por menor que seja sua intensidade de utilização no momento.

\section{Conclusões}

$\mathrm{E}$ $m$ relação aos aspectos ergonômicos, para a consecução dos resultados nos testes estáticos e dinâmicos, foi de fundamental importância a construção dos mock-ups na escala
1:1, permitindo uma avaliação bastante próxima da realidade e, principalmente, pelo fato de terem sido utilizados "sujeitos reais" na realização dos testes, o que confere ao experimento um nível de credibilidade maior do que em simulações virtuais, inclusive por permitir avaliar aspectos como níveis de conforto e, em algumas situações, o próprio comportamento dos usuários no interior do carro.

Com isso, conseguiu-se aprimorar a configuração do lay-out dos bancos e pegadores, contribuindo para o conforto dos passageiros, o aumento da capacidade dos carros e uma maior agilidade dos fluxos interno e de entrada e saída.

No tocante ao projeto do experimento, cumpre destacar a aplicação da técnica do quadrado latino às variáveis independentes de uma regressão, visando reduzir parte do experimento, no caso dos testes dinâmicos (fluxo de passageiros). Uma variável fictícia foi também introduzida, buscando-se avaliar o efeito de um fator qualitativo.

Apesar de algumas dificuldades práticas encontradas durante a sua realização, o experimento cumpriu plenamente a sua finalidade, fornecendo à Cia. do Metrô subsídios para a melhor decisão quanto à configuração do carro, bem como para futuros estudos de capacidade.

\section{Referências Bibliográficas}

COSTA NETO, P.L.O. Estatística. 2.ed. São Paulo: Edgard Blucher, 2001.

COUTO, H.A. Ergonomia - Manual da Máquina Humana. Belo Horizonte: Ergo, 1966.

DIFFRENT, N.; TILLEY, A.; BARDAGJY, J.C. Humanscale 1/2/3. Cambridge, Massachusetss: MIT-Press, 1975.

MCT - INSTITUTO NACIONAL DE TECNOLOGIA. Pesquisa Antropométrica e Biomecânica dos Operários da Indústria de Transformação - RJ - $1^{\mathrm{o}}$ volume, Medidas para Postos de Trabalho. Rio de Janeiro, 1988.
MONTGOMERY, D.C. Design and Analysis of Experiments. 3.ed. John Wiley, 1991.

PANERO, J.; ZELNIK, M. Human Dimenson \& Interior Space. New York: Whitney Library of Design, 1979.

WISNER, A. Por Dentro do Trabalho. Ergonomia - Método \& Técnica. São Paulo: FTD, 1987. 


\title{
ERGONOMICAL AND STATISTICAL ASPECTS IN THE DESIGN OF A SUBWAY CARRIAGE
}

\begin{abstract}
This paper describes experiments held as part of a project including features of the design of a carriage for a new line of the São Paulo City Subway System. It includes ergonomical aspects, related to the position of safety bars and seats, and statistical aspects, related to the flow of passengers from and to the carriage at the station. In this study, the use of the latin square procedure in multiple regression analysis is of particular interest being a way to reduce the size of the experiment.
\end{abstract}

Key words: ergonomics, antropometric measures, passengers flow, latin squares, multiple regression analysis. 